\title{
OSCILLATION CRITERIA FOR DIFFERENTIAL EQUATIONS OF SECOND ORDER
}

\author{
A. K. Nandakumaran — S. Panigrahi \\ (Communicated by Michal Fečkan)
}

\begin{abstract}
In this article, we give sufficient condition in the form of integral inequalities to establish the oscillatory nature of non linear homogeneous differential equations of the form

$$
\left(r(t) y^{\prime}\right)^{\prime}+q(t) y^{\prime}+p(t) f(y) g\left(y^{\prime}\right)=0, \quad t \geq t_{0},
$$
\end{abstract}

where $r, q, p, f$ and $g$ are given data. We do this by separating the two cases $f$ is monotonous and non monotonous.

(C) 2009

Mathematical Institute Slovak Academy of Sciences

\section{Introduction}

Comparison theorems for solution of ordinary differential equations, distribution of zeros of solutions for large time, oscillatory behavior of zeros of solutions etc. have been extensively studied in the literature. These are very important to understand the qualitative behavior of solutions specially in the non linear equations. In this article, we wish to study the oscillatory behavior of non linear homogeneous differential equations of the form

$$
\left(r(t) y^{\prime}\right)^{\prime}+q(t) y^{\prime}+p(t) f(y) g\left(y^{\prime}\right)=0, \quad t \geq t_{0},
$$

where $r, q, p, f$ and $g$ satisfies various assumptions to be given later. In fact, we give sufficient conditions to establish the oscillatory behavior. Coming to the literature, Wo n g and B urt on [28] have been investigated the oscillatory behavior in connection with that of corresponding linear homogeneous equation

$$
y^{\prime \prime}+p(t) y=0
$$

2000 Mathematics Subject Classification: Primary $34 \mathrm{C} 10$.

Keywords: oscillation, homogeneous, non linear, disfocality, disconjugacy.

Research supported by National Board of Higher Mathematics, Department of Atomic Energy, India. 


\section{A. K. NANDAKUMARAN - S. PANIGRAHI}

where $p(t)$ is oscillatory.

In [5], Grace and L alli have considered the behavior of solution of nonlinear differential equations

$$
y^{\prime \prime}+p(t) f(y(t)) g\left(y^{\prime}(t)\right)=0, \quad t \geq t_{0},
$$

where $p \in C\left(\left[t_{0}, \infty\right),[0, \infty)\right)$ and $f, g \in C(\mathbb{R}, \mathbb{R})$ with $y f(y)>0$ for $y \neq 0$ and $g(y)>0$ for $y \neq 0$.

The stability, boundedness, and convergence to zero of all solutions of equation (1.3) have been investigated by Burton and Grimmer [1], Graef and Spikes [6], [7], Lalli [13] and Wong and Burton [28].

R o g o v c h e n k o [22], established new sufficient conditions which ensure the oscillatory character of equation (1.3). These are different from those of $\mathrm{G} \mathrm{r}$ a c e and Lalli [5] and applicable to other class of equations that are not covered by the results of [5]. All the above mentioned oscillation results require the information of $p$ on the entire half-line $\left[t_{0}, \infty\right)$.

However, from Sturm separation theorem, we see that oscillation is only an interval property, that is, if there exist a sequence of subintervals $\left[a_{i}, b_{i}\right]$ of $\left[t_{0}, \infty\right)$, as $a_{i} \rightarrow \infty$, such that for each $i$, there exists a solution of (1.2) that has at least two zeros in $\left[a_{i}, b_{i}\right]$, then every solution of (1.2) is oscillatory.

E i-S a y ed [4], established an interval criterion for oscillation of a forced second-order equation but the result is not sharp because a comparison with equation of constant co-efficient is used in the proof.

In [9], $\mathrm{H} \mathrm{u}$ a $\mathrm{ng}$ established the following interval criteria for oscillation and non oscillation of the second order linear differential equation (1.2), where $p(t) \geq 0, t \in\left[t_{0}, \infty\right)$.

THEOREM 1.1. If there exists $t_{0}>0$ such that for every $n \in \mathbb{N}$,

$$
\int_{2^{n} t_{0}}^{2^{n+1} t_{0}} p(s) \mathrm{d} s \leq \frac{\alpha_{0}}{2^{n+1} t_{0}},
$$

then every solution of equation (1.2) is non oscillatory, where $\alpha_{0}=3-2 \sqrt{2}$.

(ii) If there exist $t_{0}>0$ and $\alpha>\alpha_{0}$ such that for every $n \in \mathbb{N}$,

$$
\int_{2^{n} t_{0}}^{2^{n+1} t_{0}} p(s) \mathrm{d} s \geq \frac{\alpha}{2^{n} t_{0}},
$$

then every solution of equation (1.2) is oscillatory, where $\alpha_{0}=3-2 \sqrt{2}$.

As an application, $\mathrm{H}$ u a n $\mathrm{g}$ [9] obtained the following corollary. 


\section{Corollary 1.2 .}

(i) If

$$
\lim _{t \rightarrow \infty} t \int_{t}^{2 t} p(s) \mathrm{d} s=\alpha<\frac{\alpha_{0}}{2},
$$

then every solution of equation (1.2) is non oscillatory.

(ii) If

$$
\lim _{t \rightarrow \infty} t \int_{t}^{2 t} p(s) \mathrm{d} s=\alpha>\alpha_{0},
$$

then every solution of equation (1.2) is oscillatory, where $\alpha_{0}=3-2 \sqrt{2}$.

$\mathrm{H}$ u a n g 's result do not cover the interval $\left(\frac{\alpha_{0}}{2^{n+1}} t_{0}, \frac{\alpha_{0}}{2^{n}} t_{0}\right)$, and can be seen by the following example. Take, $p(t)=\gamma / t^{2}$, with, $\gamma>0$, a constant, then

$$
\lim _{t \rightarrow \infty} t \int_{t}^{2 t} \frac{\gamma}{s^{2}}=\frac{\gamma}{2}
$$

This implies that $\mathrm{H} u$ a $\mathrm{ng}$ 's result remains open for $\gamma \in\left(\alpha_{0}, 2 \alpha_{0}\right)=(3-2 \sqrt{2}$, $6-4 \sqrt{2})$. and hence $\mathrm{H} u$ a $\mathrm{ng}$ 's oscillation criterion is not sharp. In fact, the Euler equation

$$
y^{\prime \prime}+\frac{\gamma}{t^{2}} y=0
$$

is oscillatory if $\gamma>\frac{1}{4}$, and non oscillatory if $\gamma \leq \frac{1}{4}$.

Li and A g arw a l [16], [17], [18], [19] have established new interval oscillation criteria by use of integral average technique for equation (1.3). Moreover, the mentioned oscillation results based on the information only on a sequence of subintervals of $\left[t_{0}, \infty\right)$, rather than on the whole half-line. They have shown that the obtained results are sharper than some known results which are not covered by known criteria.

We have motivated by the results of $\mathrm{R}$ a g o v chenk o [22], K ong [12] and $\mathrm{Li}$ and $\mathrm{Agarwal}$ [18]. In this paper, we consider the oscillatory behavior of nonlinear homogeneous differential equations of the form

$$
\left(r(t) y^{\prime}\right)^{\prime}+q(t) y^{\prime}+p(t) f(y) g\left(y^{\prime}\right)=0, \quad t \geq t_{0},
$$

where $r, q, p, f$ and $g$ satisfies the following conditions.

$\left(C_{1}\right) r \in C^{1}\left(\left[t_{0}, \infty\right),(0, \infty)\right), q \in C\left(\left[t_{0}, \infty\right), \mathbb{R}\right)$

$\left(C_{2}\right) p:\left[t_{0}, \infty\right) \rightarrow[0, \infty)$ is continuous and $p(t) \neq 0$ on any ray $\left[T_{0}, \infty\right)$ for some $T_{0} \geq t_{0}$. 
$\left(C_{3}\right) f: \mathbb{R} \rightarrow \mathbb{R}$ is continuous and $y f(y)>0$ for $y \neq 0$.

$\left(C_{4}\right) g: \mathbb{R} \rightarrow[L, \infty)$ is continuous, where $L>0$.

A function $y:\left[t_{0}, t_{1}\right] \rightarrow \mathbb{R}, t_{1}>t_{0}$ is called a solution of equation (1.1) on $\left[t_{0}, t_{1}\right]$ if $y(t)$ satisfies equation (1.1) for $t \in\left[t_{0}, t_{1}\right)$. In the sequel, we assume that the solution of equation (1.1) exists for any $t \geq 0$. A solution $y(t)$ of (1.1) on $\left[t_{0}, \infty\right)$ is called oscillatory if it has arbitrary large zeros, otherwise it is called non oscillatory.

The outlay of this paper is as follows. In the next Section 2, we consider the case when $f$ is monotonous. We establish a sufficient criteria in the form of an integral inequality for the oscillatory behavior of the solution of the equation (1.1). We also write down the sufficient conditions in more simplified forms in the special cases. In Section 3, we retrace all the results in Section 2, when $f$ is not necessarily monotonous. We present two examples and we prove the oscillatory behavior of the solutions using the previously established results. We also obtained oscillatory solutions of two examples numerically under different initial conditions. In final Section 4, we introduce the concepts of disfocality and disconjugacy and its connection to integral inequalities (sufficient conditions). These concepts are already in the literature (see P ar h i and P a n ig r a h i [21])

\section{Oscillation with monotonicity of $f(x)$}

In this section, we assume that

$\left(C_{5}\right) f$ is monotonous and satisfies the condition $f^{\prime}(y) \geq \mu>0$, where $\mu$ is a constant.

Definition 2.1. A function $H=H(t, s)$ belongs to a function class $X$, denoted by $H \in X$ if $H \in C\left(D, \mathbb{R}_{+}\right)$, where $D=\{(t, s):-\infty<s \leq t<\infty\}$, which satisfies

$$
H(t, t)=0, \quad H(t, s)>0 \text { for } t>s,
$$

and has partial derivatives $\frac{\partial H}{\partial t}$ and $\frac{\partial H}{\partial s}$ on $D$ such that

$$
\frac{\partial H}{\partial t}=h_{1}(t, s) H(t, s)^{1 / 2} \quad \text { and } \quad \frac{\partial H}{\partial s}=-h_{2}(t, s) H(t, s)^{1 / 2},
$$

where $h_{1}, h_{2} \in L_{l o c(D, \mathbb{R})}^{1}$.

TheOREM 2.2. Suppose that conditions $\left(C_{1}\right)-\left(C_{5}\right)$ hold.

(i) Let $y$ be a positive solution of $(1.1)$ on $[c, b)$. For any $\kappa \in C^{1}\left(\left[t_{0}, \infty\right),(0, \infty)\right)$, let

$$
u(t)=v(t) r(t)\left\{\frac{y^{\prime}(t)}{f(y(t))}+\kappa(t)\right\}, \quad t \geq t_{0},
$$


on $[c, b)$. Then for any $H \in X$,

$$
\int_{c}^{b} H(b, s) \phi(s) \mathrm{d} s \leq H(b, c) u(c)+\frac{1}{4 \mu} \int_{c}^{b} r(s) v(s)\left[h_{2}(b, s)+\frac{q(s)}{r(s)} \sqrt{H}(b, s)\right]^{2} \mathrm{~d} s .
$$

(ii) Similarly, let $y$ be a positive solution of (1.1) on $(a, c]$. For any $\kappa \in$ $C^{1}\left(\left[t_{0}, \infty\right),(0, \infty)\right)$, define $u$ as above, then for any $H \in X$

$$
\int_{a}^{c} H(s, a) \phi(s) \mathrm{d} s \leq-H(c, a) u(c)+\frac{1}{4 \mu} \int_{a}^{c} r(s) v(s)\left[h_{1}(s, a)-\frac{q(s)}{r(s)} \sqrt{H}(s, a)\right]^{2} \mathrm{~d} s .
$$

Here $v$ and $\phi$ are defined as

$$
v(t)=\exp \left(-2 \mu \int_{t_{0}}^{t} \kappa(s) \mathrm{d} s\right)
$$

and

$$
\phi(t)=v(t)\left\{L p(t)-q(t) \kappa(t)+\mu r(t) \kappa^{2}(t)-(r(t) \kappa(t))^{\prime}\right\} .
$$

P r o of. From the equations (1.1) and (2.3) for $s \in[c, b)$, we have

$$
\begin{aligned}
u^{\prime}(t)= & -2 \mu \kappa(t) v(t) r(t)\left\{\frac{y^{\prime}(t)}{f(y(t))}+\kappa(t)\right\} \\
& +v(t)\left\{\frac{\left(r(t) y^{\prime}\right)^{\prime}}{f(y(t))}-\frac{r(t) f^{\prime}(y(t)) y^{\prime 2}(t)}{f^{2}(y(t))}+(r(t) \kappa(t))^{\prime}\right\}
\end{aligned}
$$

By using $f^{\prime}(y) \geq \mu>0$ and $g\left(y^{\prime}(t)\right) \geq L>0$, we obtain from the above inequality

$$
\begin{aligned}
u^{\prime}(t) \leq- & 2 \mu \kappa(t) v(t) r(t) \frac{y^{\prime}(t)}{f(y(t))}-2 \mu \kappa^{2}(t) v(t) r(t)-\frac{q(t) v(t) y^{\prime}(t)}{f(y(t))} \\
& -L v(t) p(t)-\frac{\mu r(t) v(t) y^{\prime 2}(t)}{f^{2}(y(t))}+v(t)(r(t) \kappa(t))^{\prime} \\
= & -\frac{\mu u^{2}(t)}{r(t) v(t)}-\frac{q(t)}{r(t)} u(t)-v(t)\left[L p(t)-q(t) \kappa(t)+\mu r(t) \kappa^{2}(t)-(r(t) \kappa(t))^{\prime}\right] \\
= & -\frac{\mu u^{2}(t)}{r(t) v(t)}-\frac{q(t)}{r(t)} u(t)-\phi(t) .
\end{aligned}
$$

Thus

$$
\phi(t) \leq-u^{\prime}(t)-\frac{\mu u^{2}(t)}{r(t) v(t)}-\frac{q(t)}{r(t)} u(t)
$$


Multiplying (2.6) by $H(t, s)$, integrating it with respect to $s$ from $c$ to $t(c \leq$ $t<b)$, and using (2.1) and (2.2), we get

$$
\begin{aligned}
& \int_{c}^{t} H(t, s) \phi(s) \mathrm{d} s \\
\leq & -\int_{c}^{t} H(t, s) u^{\prime}(s) \mathrm{d} s-\int_{c}^{t} H(t, s) \frac{\mu u^{2}(s)}{r(s) v(s)} \mathrm{d} s-\int_{c}^{t} H(t, s) \frac{q(s)}{r(s)} u(s) \mathrm{d} s \\
= & H(t, c) u(c)-\int_{c}^{t}\left\{h_{2}(t, s) \sqrt{H}(t, s) u(s)+H(t, s) \frac{q(s)}{r(s)} u(s)+H(t, s) \frac{\mu u^{2}(s)}{r(s) v(s)}\right\} \mathrm{d} s \\
= & H(t, c) u(c)-\int_{c}^{t}\left\{\sqrt{\frac{\mu H(t, s)}{r(s) v(s)}} u(s)+\frac{1}{2} \sqrt{\frac{r(s) v(s)}{\mu}}\left[h_{2}(t, s)+\frac{q(s)}{r(s)} \sqrt{H}(t, s)\right]\right\}^{2} \mathrm{~d} s \\
& +\frac{1}{4 \mu} \int_{c}^{t} r(s) v(s)\left[h_{2}(t, s)+\frac{q(s)}{r(s)} \sqrt{H}(t, s)\right]^{2} \mathrm{~d} s \\
\leq & H(t, c) u(c)+\frac{1}{4 \mu} \int_{c}^{t} r(s) v(s)\left[h_{2}(t, s)+\frac{q(s)}{r(s)} \sqrt{H}(t, s)\right]^{2} \mathrm{~d} s .
\end{aligned}
$$

Taking $t \rightarrow b^{-}$, in the above inequality, we obtain (2.4).

To get the second part, again multiply $(2.6)$ by $H(s, t)$, integrate with respect to $s$ from $t$ to $c$ for $t \in(a, c]$. Using (2.1) and (2.2), we get

$$
\begin{aligned}
& \int_{t}^{c} H(s, t) \phi(s) \mathrm{d} s \\
\leq & -\int_{t}^{c} H(s, t) u^{\prime}(s) \mathrm{d} s-\int_{t}^{c} H(s, t) \frac{\mu u^{2}(s)}{r(s) v(s)} \mathrm{d} s-\int_{t}^{c} H(s, t) \frac{q(s)}{r(s)} u(s) \mathrm{d} s \\
= & H(c, t) u(c)+\int_{t}^{c}\left\{h_{1}(s, t) \sqrt{H}(s, t) u(s)-H(s, t) \frac{q(s)}{r(s)} u(s)-H(s, t) \frac{\mu u^{2}(s)}{r(s) v(s)}\right\} \mathrm{d} s \\
= & H(c, t) u(c)-\int_{t}^{c}\left\{\sqrt{\frac{\mu H(s, t)}{r(s) v(s)}} u(s)-\frac{1}{2} \sqrt{\frac{r(s) v(s)}{\mu}}\left[h_{1}(s, t)-\frac{q(s)}{r(s)} \sqrt{H}(s, t)\right]\right\}^{2} \mathrm{~d} s \\
& +\frac{1}{4 \mu} \int_{t}^{c} r(s) v(s)\left[h_{1}(s, t)-\frac{q(s)}{r(s)} \sqrt{H}(s, t)\right]^{2} \mathrm{~d} s \\
\leq & H(c, t) u(c)+\frac{1}{4 \mu} \int_{t}^{c} r(s) v(s)\left[h_{1}(s, t)-\frac{q(s)}{r(s)} \sqrt{H}(s, t)\right]^{2} \mathrm{~d} s .
\end{aligned}
$$




\section{OSCILLATION CRITERIA FOR DIFFERENTIAL EQUATIONS OF SECOND ORDER}

Taking $t \rightarrow a^{+}$, in the above inequality, we obtain (2.5). The proof of the theorem is complete.

Theorem 2.3. Suppose that $\left(C_{1}\right)-\left(C_{5}\right)$ hold. Assume that for some $c \in(a, b)$, there exist $H \in X, \kappa \in C^{1}\left(\left[t_{0}, \infty\right),(0, \infty)\right)$, the following inequality is satisfied

$$
\begin{gathered}
\frac{1}{H(c, a)} \int_{a}^{c} H(s, a) \phi(s) \mathrm{d} s+\frac{1}{H(b, c)} \int_{c}^{b} H(b, s) \phi(s) \mathrm{d} s \\
>\frac{1}{4 \mu H(c, a)} \int_{a}^{c} r(s) v(s)\left[h_{1}(s, a)-\frac{q(s)}{r(s)} \sqrt{H}(s, a)\right]^{2} \mathrm{~d} s \\
+\frac{1}{4 \mu H(b, c)} \int_{c}^{b} r(s) v(s)\left[h_{2}(b, s)+\frac{q(s)}{r(s)} \sqrt{H}(b, s)\right]^{2} \mathrm{~d} s,
\end{gathered}
$$

where $u, v, \phi, h_{1}, h_{2}$ are defined as earlier. Then every solution of (1.1) has at least one zero in $(a, b)$.

P r o of. If not, then with out loss of generality we may assume that equation (1.1) admits a solution $y(t)$ such that $y(t)>0$ for $t \in(a, b)$. Let $c \in(a, b)$. Then by using Theorem 2.2, we conclude that (2.4) and (2.5) holds. By dividing (2.4) and $(2.5)$ by $H(b, c)$ and $H(c, a)$ respectively, and adding them, we have

$$
\begin{aligned}
& \frac{1}{H(c, a)} \int_{a}^{c} H(s, a) \phi(s) \mathrm{d} s+\frac{1}{H(b, c)} \int_{c}^{b} H(b, s) \phi(s) \mathrm{d} s \\
& \leq \frac{1}{4 \mu H(c, a)} \int_{a}^{c} r(s) v(s)\left[h_{1}(s, a)-\frac{q(s)}{r(s)} \sqrt{H}(s, a)\right]^{2} \mathrm{~d} s \\
& +\frac{1}{4 \mu H(b, c)} \int_{c}^{b} r(s) v(s)\left[h_{2}(b, s)+\frac{q(s)}{r(s)} \sqrt{H}(b, s)\right]^{2} \mathrm{~d} s,
\end{aligned}
$$

which is a contradiction to (2.7). Hence the proof of the theorem is complete.

\section{Theorems on oscillations}

We now derive various oscillatory criteria using the earlier results.

TheOREM 2.4. Suppose that $\left(C_{1}\right)-\left(C_{5}\right)$ hold. If for each $T \geq t_{0}$, there exist $H \in X, \kappa \in C^{1}\left(\left[t_{0}, \infty\right),(0, \infty)\right)$ and $a, b, c \in \mathbb{R}$ such that $T \leq a<c<b$ and (2.7) holds, then every solution of (1.1) is oscillatory. 


\section{A. K. NANDAKUMARAN - S. PANIGRAHI}

Proof. We consider a sequence $\left\{T_{i}\right\} \subset\left[t_{0}, \infty\right)$ such that $T_{i} \rightarrow \infty$ as $i \rightarrow \infty$. By the assumption, for each $i \in \mathbb{N}$, there exists $a_{i}, b_{i}, c_{i}$ and such that $T \leq a_{i}<$ $c_{i}<b_{i}$ and (2.7) holds. From Theorem 2.3, every solution $y(t)$ has at least one zero in $\left(a_{i}, b_{i}\right)$. As $T_{i} \rightarrow \infty$, we see that every solution of (1.1) is oscillatory. Hence the theorem.

TheOREM 2.5. Suppose that conditions $\left(C_{1}\right)-\left(C_{5}\right)$ hold. Assume there exist $H \in X, \kappa \in C^{1}\left(\left[t_{0}, \infty\right),(0, \infty)\right)$ such that for any $t_{1} \geq t_{0}$,

$$
\limsup _{t \rightarrow \infty} \int_{t_{1}}^{t}\left\{H\left(s, t_{1}\right) \phi(s)-\frac{1}{4 \mu} r(s) v(s)\left[h_{1}\left(s, t_{1}\right)-\frac{q(s)}{r(s)} \sqrt{H}\left(s, t_{1}\right)\right]^{2}\right\} \mathrm{d} s>0
$$

and

$$
\limsup _{t \rightarrow \infty} \int_{t_{1}}^{t}\left\{H(t, s) \phi(s)-\frac{1}{4 \mu} r(s) v(s)\left[h_{2}(t, s)+\frac{q(s)}{r(s)} \sqrt{H}(t, s)\right]^{2}\right\} \mathrm{d} s>0
$$

where $v(t)$ and $\phi(t)$ are defined as in Theorem 2.1. Then every solution of (1.1) is oscillatory.

Proof. For any $T \geq t_{0}$, let $a=T$. In (2.8), take $t_{1}=a$. Then there exists $c>a$ such that

$$
\int_{a}^{c}\left\{H(s, a) \phi(s)-\frac{1}{4 \mu} r(s) v(s)\left[h_{1}(s, a)-\frac{q(s)}{r(s)} \sqrt{H}(s, a)\right]^{2}\right\} \mathrm{d} s>0 .
$$

In $(2.9)$, take $t_{1}=c$. Then there exist $b>c$ such that

$$
\int_{c}^{b}\left\{H(b, s) \phi(s)-\frac{1}{4 \mu} r(s) v(s)\left[h_{2}(b, s)+\frac{q(s)}{r(s)} \sqrt{H}(b, s)\right]^{2}\right\} \mathrm{d} s>0 .
$$




\section{OSCILLATION CRITERIA FOR DIFFERENTIAL EQUATIONS OF SECOND ORDER}

Dividing the equations (2.10) and (2.11), by $H(c, a)$ and $H(b, c)$, respectively, and adding, we get

$$
\begin{aligned}
& \frac{1}{H(c, a)} \int_{a}^{c} H(s, a) \phi(s) \mathrm{d} s+\frac{1}{H(b, c)} \int_{c}^{b} H(b, s) \phi(s) \mathrm{d} s \\
> & \frac{1}{4 \mu H(c, a)} \int_{a}^{c} r(s) v(s)\left[h_{1}(s, a)-\frac{q(s)}{r(s)} \sqrt{H}(s, a)\right]^{2} \mathrm{~d} s \\
& +\frac{1}{4 \mu H(b, c)} \int_{c}^{b} r(s) v(s)\left[h_{2}(b, s)+\frac{q(s)}{r(s)} \sqrt{H}(b, s)\right]^{2} \mathrm{~d} s .
\end{aligned}
$$

Then it follows by Theorem 2.4, that every solution of (1.1) is oscillatory.

Remark 2.6. If $q(t)=0, f(y)=y, g\left(y^{\prime}\right)=1, \kappa(t)=0$, then Theorems 2.3 and 2.5 reduces to $[12$, Corollaries $2.2,2.4]$.

Consider the special case $H(t, s)=H(t-s)$, then

$$
\frac{\partial H}{\partial t}=h_{1}(t-s) H(t-s)^{1 / 2} \quad \text { and } \quad \frac{\partial H}{\partial s}=h_{2}(t-s) H(t-s)^{1 / 2} .
$$

Thus for $H=H(t-s) \in X$, we have $h_{1}(t-s)=h_{2}(t-s)$ and denote them by $h(t-s)$. The subclass containing such $H(t-s)$ is denoted by $X_{0}$. Applying Theorem 2.4 to $X_{0}$ we have the following result:

THEOREM 2.7. Suppose that conditions $\left(C_{1}\right)-\left(C_{5}\right)$ hold. If for each $T \geq t_{0}$, there exist $H \in X_{0}, \kappa \in C^{1}\left(\left[t_{0}, \infty\right),(0, \infty)\right)$ and $a, c \in \mathbb{R}$ such that $T \leq a<c$ and

$$
\begin{aligned}
& \int_{a}^{c} H(s-a)[\phi(s)+\phi(2 c-s)] \mathrm{d} s \\
& >\frac{1}{4 \mu} \int_{a}^{c}[r(s) v(s)+r(2 c-s) v(2 c-s)] h^{2}(s-a) \mathrm{d} s \\
& +\frac{1}{2 \mu} \int_{a}^{c}[v(2 c-s) q(2 c-s)-v(s) q(s)] h(s-a) \sqrt{H}(s-a) \mathrm{d} s \\
& +\frac{1}{4 \mu} \int_{a}^{c}\left[\frac{v(s) q^{2}(s)}{r(s)}+\frac{v(2 c-s) q^{2}(2 c-s)}{r(2 c-s)}\right] H(s-a) \mathrm{d} s,
\end{aligned}
$$

where $v(t)$ and $\phi(t)$ are as defined in Theorem 2.1, then every solution of equation (1.1) is oscillatory. 


\section{A. K. NANDAKUMARAN - S. PANIGRAHI}

Proof. Let $c=\frac{a+b}{2}$, that is, $b=2 c-a$, then

$$
H(b-c)=H(c-a)=H((b-a) / 2) .
$$

For any $\omega \in L^{1}(a, b)$, we have

$$
\begin{aligned}
\int_{c}^{b} \omega(s) \mathrm{d} s & =\int_{a}^{c} \omega(2 c-s) \mathrm{d} s \\
\int_{c}^{b} H(b-s) \phi(s) \mathrm{d} s & =\int_{a}^{c} H(s-a) \phi(2 c-s) \mathrm{d} s \\
\int_{c}^{b} v(s) q(s) h(b-s) \sqrt{H}(b-s) \mathrm{d} s & =\int_{a}^{c} v(2 c-s) q(2 c-s) h(s-a) \sqrt{H}(s-a) \mathrm{d} s \\
\int_{c}^{b} r(s) v(s) h^{2}(b-s) \mathrm{d} s & =\int_{a}^{c} r(2 c-s) v(2 c-s) h^{2}(s-a) \sqrt{H}(s-a) \mathrm{d} s .
\end{aligned}
$$

It is easy to see that (2.12) implies that the inequality (2.7). Hence every solution of (1.1) is oscillatory by Theorem 2.4.

For different choices of $H(t, s)$, we will obtain different sufficient conditions for oscillatory behavior of solutions of equation (1.1). Let

$$
H(t, s)=(t-s)^{\lambda}, \quad t \geq s \geq t_{0},
$$

where $\lambda>1$ is a constant. Then the sufficient conditions (2.8) and (2.9), respectively can be written as

$$
\limsup _{t \rightarrow \infty} \int_{t_{1}}^{t}\left(s-t_{1}\right)^{\lambda}\left[\phi(s)-\frac{1}{4 \mu} r(s) v(s)\left(\frac{\lambda}{\left(s-t_{1}\right)}-\frac{q(s)}{r(s)}\right)^{2}\right] \mathrm{d} s>0,
$$

and

$$
\limsup _{t \rightarrow \infty} \int_{t_{1}}^{t}(t-s)^{\lambda}\left[\phi(s)-\frac{1}{4 \mu} r(s) v(s)\left(\frac{\lambda}{(t-s)}+\frac{q(s)}{r(s)}\right)^{2}\right] \mathrm{d} s>0 .
$$

Corollary 2.8. Suppose that $\left(C_{1}\right)-\left(C_{5}\right)$ hold. Assume for each $t_{1} \geq t_{0}$ and for some $\lambda>1, \kappa \in C^{1}\left(\left[t_{0}, \infty\right),(0, \infty)\right)$, we have

$$
\limsup _{t \rightarrow \infty} \frac{1}{t^{\lambda-1}} \int_{t_{1}}^{t}\left(s-t_{1}\right)^{\lambda}\left[\phi(s)-\frac{1}{4 \mu} r(s) v(s)\left(\frac{\lambda}{\left(s-t_{1}\right)}-\frac{q(s)}{r(s)}\right)^{2}\right] \mathrm{d} s>0,
$$


and

$$
\limsup _{t \rightarrow \infty} \frac{1}{t^{\lambda-1}} \int_{t_{1}}^{t}(t-s)^{\lambda}\left[\phi(s)-\frac{1}{4 \mu} r(s) v(s)\left(\frac{\lambda}{(t-s)}+\frac{q(s)}{r(s)}\right)^{2}\right] \mathrm{d} s>0,
$$

then every solution of (1.1) is oscillatory.

The oscillatory nature of the solutions follows because, the above two conditions implies the conditions (2.13) and (2.14). We, however use the above two conditions to get the following result.

TheOREM 2.9. Suppose that $\left(C_{1}\right)-\left(C_{5}\right)$ hold. If for each $t_{1} \geq t_{0}$ and for some $\lambda>1$ satisfies the following conditions

$$
\limsup _{t \rightarrow \infty} \frac{1}{t^{\lambda-1}} \int_{t_{1}}^{t}\left(s-t_{1}\right)^{\lambda}\left[\phi(s)-\frac{1}{4 \mu}\left(q^{2}(s)-\frac{2 \lambda q(s)}{\left(s-t_{1}\right)}\right)\right] \mathrm{d} s>\frac{\lambda^{2}}{4 \mu(\lambda-1)}
$$

and

$$
\limsup _{t \rightarrow \infty} \frac{1}{t^{\lambda-1}} \int_{t_{1}}^{t}(t-s)^{\lambda}\left[\phi(s)-\frac{1}{4 \mu}\left(q^{2}(s)+\frac{2 \lambda q(s)}{(t-s)}\right)\right] \mathrm{d} s>\frac{\lambda^{2}}{4 \mu(\lambda-1)},
$$

then (1.1) is oscillatory.

Proof. We may observe that

$$
h_{1}(t, s)=h_{2}(t, s)=\lambda(t-s)^{\lambda / 2-1} .
$$

Note that

$$
\begin{aligned}
\limsup _{t \rightarrow \infty} \frac{1}{4 \mu t^{\lambda-1}} \int_{t_{1}}^{t} h_{1}^{2}\left(s-t_{1}\right) \mathrm{d} s & =\limsup _{t \rightarrow \infty} \frac{1}{4 \mu t^{\lambda-1}} \int_{t_{1}}^{t} \lambda^{2}\left(s-t_{1}\right)^{\lambda-2} \mathrm{~d} s \\
& =\frac{\lambda^{2}}{4 \mu(\lambda-1)}
\end{aligned}
$$

and

$$
\begin{aligned}
\limsup _{t \rightarrow \infty} \frac{1}{4 \mu t^{\lambda-1}} \int_{t_{1}}^{t} h_{2}^{2}(t-s) \mathrm{d} s & =\limsup _{t \rightarrow \infty} \frac{1}{4 \mu t^{\lambda-1}} \int_{t_{1}}^{t} \lambda^{2}(t-s)^{\lambda-2} \mathrm{~d} s \\
& =\frac{\lambda^{2}}{4 \mu(\lambda-1)}
\end{aligned}
$$




\section{A. K. NANDAKUMARAN - S. PANIGRAHI}

From (2.15) and (2.17), we have

$$
\begin{aligned}
& \limsup _{t \rightarrow \infty} \frac{1}{t^{\lambda-1}} \int_{t_{1}}^{t}\left\{H\left(s-t_{1}\right) \phi(s)-\frac{1}{4 \mu}\left[h_{1}\left(s-t_{1}\right)-q(s) \sqrt{H}\left(s-t_{1}\right)\right]^{2}\right\} \mathrm{d} s \\
= & \limsup _{t \rightarrow \infty} \frac{1}{t^{\lambda-1}} \int_{t_{1}}^{t}\left(s-t_{1}\right)^{\lambda}\left[\phi(s)-\frac{1}{4 \mu}\left(q^{2}(s)+\frac{2 \lambda q(s)}{\left(s-t_{1}\right)}\right)\right] \mathrm{d} s-\frac{\lambda^{2}}{4 \mu(\lambda-1)}>0,
\end{aligned}
$$

that is, (2.8) holds. Similarly, (2.16) and (2.18) implies that (2.9) holds. By Theorem 2.5, every solution of (1.1) is oscillatory. Hence the theorem.

More generally, one may consider

$$
H(t, s)=[R(t)-R(s)]^{\lambda},
$$

where $\lambda$ is a constant and $R(t)=\int_{t_{1}}^{t} \frac{1}{r(s)} \mathrm{d} s$ and $\lim _{t \rightarrow \infty} R(t)=\infty$.

Theorem 2.10. Suppose that $\left(C_{1}\right)-\left(C_{5}\right)$ hold. If for each $t_{1} \geq t_{0}$ and for some $\left.\lambda>1, \kappa \in C^{1}\left(\left[t_{0}\right), \infty\right),(0, \infty)\right)$ such that

$$
\limsup _{t \rightarrow \infty} \frac{1}{R^{\lambda-1}(t)} \int_{t_{1}}^{t}\left[R(s)-R\left(t_{1}\right)\right]^{\lambda}\left[\phi(s)-\frac{v(s)}{4 \mu r(s)}\left(\frac{\lambda}{R(s)-R\left(t_{1}\right)}-q(s)\right)^{2}\right] \mathrm{d} s>0,
$$

and

$$
\limsup _{t \rightarrow \infty} \frac{1}{R^{\lambda-1}(t)} \int_{t_{1}}^{t}[R(t)-R(s)]^{\lambda}\left[\phi(s)-\frac{v(s)}{4 \mu r(s)}\left(\frac{\lambda}{R(t)-R(s)}+q(s)\right)^{2}\right] \mathrm{d} s>0,
$$

then every solution of (1.1) is oscillatory.

The proof is similar to that of Theorem 2.5 and hence omitted. We also have the following theorem whose proof is similar to Theorem 2.9.

TheOREM 2.11. Suppose that $\left(C_{1}\right)-\left(C_{5}\right)$ hold. If for each $t_{1} \geq t_{0}$ and for some $\lambda>1, \kappa \in C^{1}\left(\left[t_{0}, \infty\right),(0, \infty)\right)$ such that

$$
\begin{array}{r}
\limsup _{t \rightarrow \infty} \frac{1}{R^{\lambda-1}(t)} \int_{t_{1}}^{t}\left[R(s)-R\left(t_{1}\right)\right]^{\lambda}\left[\phi(s)-\frac{1}{4 \mu r(s)}\left(q^{2}(s)-\frac{2 \lambda q(s)}{R(s)-R\left(t_{1}\right)}\right)\right] \mathrm{d} s \\
>\frac{\lambda^{2}}{4 \mu\left(\lambda^{2}-1\right)}
\end{array}
$$


and

$$
\begin{array}{r}
\limsup _{t \rightarrow \infty} \frac{1}{R^{\lambda-1}(t)} \int_{t_{1}}^{t}[R(t)-R(s)]^{\lambda}\left[\phi(s)-\frac{1}{4 \mu r(s)}\left(q^{2}(s)+\frac{2 \lambda q(s)}{R(t)-R(s)}\right)\right] \mathrm{d} s \\
>\frac{\lambda^{2}}{4 \mu\left(\lambda^{2}-1\right)},
\end{array}
$$

then every solution of (1.1) is oscillatory.

\section{Oscillation without monotonicity of $f(x)$}

We now consider non monotonous situation. But, we make the following assumption

$\left(C_{6}\right) \frac{f(y)}{y} \geq \mu_{1}>0$, where $\mu_{1}$ is a constant.

TheOREM 3.1. Suppose that conditions $\left(C_{1}\right)-\left(C_{4}\right)$ and $\left(C_{6}\right)$ hold.

(i) Let $y$ be a solution of (1.1) such that $y(t)>0$ on $[c, b)$. For any $\kappa \in$ $C^{1}\left(\left[t_{0}, \infty\right),(0, \infty)\right)$, let

$$
\omega(t)=v(t) r(t)\left\{\frac{y^{\prime}(t)}{y(t)}+\kappa(t)\right\}, \quad t \geq t_{0},
$$

on $[c, b)$. Then for any $H \in X$, we have

$$
\int_{c}^{b} H(t, s) \phi(s) \mathrm{d} s \leq H(b, c) \omega(c)+\frac{1}{4} \int_{c}^{b} r(s) v(s)\left[h_{2}(b, c)+\frac{q(s)}{r(s)} \sqrt{H}(b, c)\right]^{2} \mathrm{~d} s .
$$

(ii) Let $y$ be a solution of (1.1) such that $y(t)>0$ on $(a, c]$ and $\omega$ be as above. Then for any $H \in X$, we have

$$
\int_{a}^{c} H(s, a) \phi(s) \mathrm{d} s \leq-H(c, a) \omega(c)+\frac{1}{4} \int_{a}^{c} r(s) v(s)\left[h_{1}(s, a)+\frac{q(s)}{r(s)} \sqrt{H}(s, a)\right]^{2} \mathrm{~d} s,
$$

where

$$
v(t)=\exp \left(-2 \int_{t_{0}}^{t} \kappa(s) \mathrm{d} s\right)
$$

and

$$
\phi(t)=v(t)\left\{L \mu_{1} p(t)-q(t) \kappa(t)+r(t) \kappa^{2}(t)-(r(t) \kappa(t))^{\prime}\right\} .
$$




\section{A. K. NANDAKUMARAN — S. PANIGRAHI}

P r o of. From (1.1) and (3.1), we have

$$
\begin{aligned}
\omega^{\prime}(t)=- & \kappa(t) v(t) r(t)\left\{\frac{y^{\prime}(t)}{y(t)}+\kappa(t)\right\} \\
& +v(t)\left\{\frac{\left(r(t) y^{\prime}\right)^{\prime}}{y(t)}-\frac{r(t) y^{\prime 2}(t)}{y^{2}(t)}+(r(t) \kappa(t))^{\prime}\right\} .
\end{aligned}
$$

By using $f(y) y \geq \mu_{1}>0$ and $g\left(y^{\prime}(t)\right) \geq L>0$, we obtain from the above inequality

$$
\begin{aligned}
& \omega^{\prime}(t) \leq-2 \kappa(t) v(t) r(t) \frac{y^{\prime}(t)}{y(t)}-2 \kappa^{2}(t) v(t) r(t)-q(t) v(t) \frac{y^{\prime}(t)}{y(t)} \\
& -L \mu_{1} v(t) p(t)-r(t) v(t) \frac{y^{\prime 2}(t)}{y^{2}(t)}+v(t)(r(t) \kappa(t))^{\prime} \\
= & -\frac{\omega^{2}(t)}{r(t) v(t)}-\frac{q(t)}{r(t)} \omega(t)-v(t)\left[L \mu_{1} p(t)-q(t) \kappa(t)+r(t) \kappa^{2}(t)-(r(t) \kappa(t))^{\prime}\right] \\
= & -\frac{\omega^{2}(t)}{r(t) v(t)}-\frac{q(t)}{r(t)} \omega(t)-\phi(t)
\end{aligned}
$$

that is,

$$
\phi(t) \leq-\omega^{\prime}(t)-\frac{\omega^{2}(t)}{r(t) v(t)}-\frac{q(t)}{r(t)} \omega(t)
$$

The remaining part of the proof is the same as that of the proof of Theorem 2.1 and hence omitted.

Once we have the above theorem, we obtain similar results as in Section 2. For the sake of completeness, we present the results without proof.

TheOrem 3.2. Suppose that $\left(C_{1}\right)-\left(C_{4}\right)$ and $\left(C_{6}\right)$ hold. Assume that for some $c \in(a, b), H \in X, \kappa \in C^{1}\left(\left[t_{0}, \infty\right),(0, \infty)\right)$, the following inequality is satisfied

$$
\begin{aligned}
& \frac{1}{H(c, a)} \int_{a}^{c} H(s, a) \phi(s) \mathrm{d} s+\frac{1}{H(b, c)} \int_{c}^{b} H(b, s) \phi(s) \mathrm{d} s \\
> & \frac{1}{4 H(c, a)} \int_{a}^{c} r(s) v(s)\left[h_{1}(s, a)-\frac{q(s)}{r(s)} \sqrt{H}(s, a)\right]^{2} \mathrm{~d} s \\
& +\frac{1}{4 H(b, c)} \int_{c}^{b} r(s) v(s)\left[h_{2}(b, s)+\frac{q(s)}{r(s)} \sqrt{H}(b, s)\right]^{2} \mathrm{~d} s .
\end{aligned}
$$

Then every solution of (1.1) has at least one zero in $(a, b)$. 


\section{OSCILLATION CRITERIA FOR DIFFERENTIAL EQUATIONS OF SECOND ORDER}

THEOREM 3.3. Suppose that conditions $\left(C_{1}\right)-\left(C_{4}\right)$ and $\left(C_{6}\right)$ hold. If for each $T \geq t_{0}$, there exist $H \in X, \kappa \in C^{1}\left(\left[t_{0}, \infty\right),(0, \infty)\right)$ and $a, b, c \in \mathbb{R}$ such that $T \leq a<c<b$ and (3.2) holds, then every solution of (1.1) is oscillatory.

Theorem 3.4. Suppose that conditions $\left(C_{1}\right)-\left(C_{4}\right)$ and $\left(C_{6}\right)$ hold. Assume there exist $H \in X, \kappa \in C^{1}\left(\left[t_{0}, \infty\right),(0, \infty)\right)$ such that for $t_{1} \geq t_{0}$,

$$
\limsup _{t \rightarrow \infty} \int_{t_{1}}^{t}\left\{H\left(s, t_{1}\right) \phi(s)-\frac{1}{4} r(s) v(s)\left[h_{1}\left(s, t_{1}\right)-\frac{q(s)}{r(s)} \sqrt{H}\left(s, t_{1}\right)\right]^{2}\right\} \mathrm{d} s>0
$$

and

$$
\limsup _{t \rightarrow \infty} \int_{t_{1}}^{t}\left\{H(t, s) \phi(s)-\frac{1}{4} r(s) v(s)\left[h_{2}(t, s)+\frac{q(s)}{r(s)} \sqrt{H}(t, s)\right]^{2}\right\} \mathrm{d} s>0,
$$

then every solution of (1.1) is oscillatory.

Corollary 3.5. Suppose that conditions $\left(C_{1}\right)-\left(C_{4}\right)$ and $\left(C_{6}\right)$ hold. If for each $T \geq t_{0}$ and for some $\lambda>1$, then there exists a function $H \in X, \kappa \in$ $C^{1}\left(\left[t_{0}, \infty\right),(0, \infty)\right)$, such that

$$
\limsup _{t \rightarrow \infty} \frac{1}{t^{\lambda-1}} \int_{t_{1}}^{t}\left(s-t_{1}\right)^{\lambda}\left[\phi(s)-\frac{1}{4} r(s) v(s)\left(\frac{\lambda}{\left(s-t_{1}\right)}-\frac{q(s)}{r(s)}\right)^{2}\right] \mathrm{d} s>0
$$

and

$$
\limsup _{t \rightarrow \infty} \frac{1}{t^{\lambda-1}} \int_{t_{1}}^{t}(t-s)^{\lambda}\left[\phi(s)-\frac{1}{4} r(s) v(s)\left(\frac{\lambda}{(t-s)}-\frac{q(s)}{r(s)}\right)^{2}\right] \mathrm{d} s>0
$$

Then every solution of (1.1) is oscillatory.

We complete this section by presenting two examples. We conclude the oscillatory nature of the solutions by employing the previous results.

Example 3.6. Consider the nonlinear homogeneous differential equations

$$
\left(t^{2} y^{\prime}\right)^{\prime}-2 t y^{\prime}+\frac{t^{2}}{\left(1+\sin ^{2} t\right)\left(1+\cos ^{4} t\right)} y\left(1+y^{4}\right)\left(1+y^{\prime 2}\right)=0, \quad t \geq 1 .
$$

Here $r(t)=t^{2}, q(t)=-2 t, p(t)=\frac{t^{2}}{\left(1+\sin ^{2} t\right)\left(1+\cos ^{4} t\right)}, f(y)=y\left(1+y^{4}\right)$ and $g\left(y^{\prime}\right)=1+y^{\prime 2}$. We may observe that

$$
f^{\prime}(y)=1+5 y^{4} \geq 1=\mu, \quad \text { and } \quad g\left(y^{\prime}\right)=1+y^{\prime 2} \geq 1=L .
$$

If $\lambda=2$ and we choose $v(t)=\exp \left(-2 \mu \int_{1}^{t} \kappa(s)\right) \mathrm{d} s=\frac{1}{t^{2}}$, then $\phi(t)=v(t)\{L p(t)-$ $\left.\kappa(t) q(t)+\mu r(t) \kappa^{2}(t)-(r(t) \kappa(t))^{\prime}\right\}=\frac{1}{\left(1+\sin ^{2} t\right)\left(1+\cos ^{4} t\right)}+2$. 


\section{A. K. NANDAKUMARAN - S. PANIGRAHI}

By use of Corollary 2.8, we may show that

$$
\begin{aligned}
& \limsup _{t \rightarrow \infty} \frac{1}{t^{\lambda-1}} \int_{t_{1}}^{t}\left(s-t_{1}\right)^{\lambda}\left[\phi(s)-\frac{1}{4 \mu} r(s) v(s)\left(\frac{\lambda}{s-t_{1}}-\frac{q(s)}{r(s)}\right)^{2}\right] \mathrm{d} s \\
= & \limsup _{t \rightarrow \infty} \frac{1}{t} \int_{t_{1}}^{t}\left(s-t_{1}\right)^{2}\left[\frac{1}{\left(1+\sin ^{2} s\right)\left(1+\cos ^{4} s\right)}+2-\frac{1}{2}\left(\frac{1}{s-t_{1}}+\frac{1}{s}\right)^{2}\right] \mathrm{d} s \\
\geq & \limsup _{t \rightarrow \infty} \frac{1}{t} \int_{t_{1}}^{t}\left[\left(s-t_{1}\right)^{2}-\frac{1}{2 s^{2}}\left(2 s-t_{1}\right)^{2}+2\right] \mathrm{d} s=\infty,
\end{aligned}
$$

and

$$
\begin{aligned}
& \limsup _{t \rightarrow \infty} \frac{1}{t^{\lambda-1}} \int_{t_{1}}^{t}(t-s)^{\lambda}\left[\phi(s)-\frac{1}{4 \mu} r(s) v(s)\left(\frac{\lambda}{t-s}-\frac{q(s)}{r(s)}\right)^{2}\right] \mathrm{d} s \\
= & \limsup _{t \rightarrow \infty} \frac{1}{t} \int_{t_{1}}^{t}(t-s)^{2}\left[\frac{1}{\left(1+\sin ^{2} s\right)\left(1+\cos ^{4} s\right)}+2-\left(\frac{1}{t-s}+\frac{1}{s}\right)^{2}\right] \mathrm{d} s=\infty .
\end{aligned}
$$

Hence (3.3) is oscillatory by Corollary 1. Moreover, $y=\cos t$ is an oscillatory solution of (3.3).

Example 3.7. Consider the nonlinear homogeneous differential equation

$$
\left(t y^{\prime}\right)^{\prime}-y^{\prime}+\frac{3 t\left(1+\sin ^{2} t\right)}{\left(1+\cos ^{2} t\right)\left(4+\sin ^{2} t\right)} y\left(\frac{1}{3}+\frac{1}{1+y^{2}}\right)\left(1+y^{\prime 2}\right)=0, \quad t \geq 1 .
$$

Here $r(t)=t, q(t)=-1, p(t)=\frac{3 t\left(1+\sin ^{2} t\right)}{\left(4+\sin ^{2} t\right)\left(1+\cos ^{2} t\right)}, f(y)=\left(\frac{1}{3}+\frac{1}{1+y^{2}}\right) y, g\left(y^{\prime}\right)=$ $1+y^{\prime 2} \geq 1=L$. As earlier, we take $\lambda=2$ and $v(t)=\frac{1}{t^{2}}$. We may observe that

$$
\begin{aligned}
f^{\prime}(y) & =\frac{y^{4}-y^{2}+4}{\left(1+y^{2}\right)^{2}} \\
\frac{f(y)}{y} & =\frac{1}{3}+\frac{1}{1+y^{2}} \geq \frac{1}{3}=\mu_{1} \\
g\left(y^{\prime}\right) & =1+y^{\prime 2} \geq 1=L .
\end{aligned}
$$

Thus

$$
\begin{aligned}
\phi(t) & =v(t)\left[L \mu_{1} p(t)-\kappa(t) q(t)+r(t) \kappa^{2}(t)-(r(t) \kappa(t))^{\prime}\right] \\
& =\frac{1+\sin ^{2} t}{t\left(4+\sin ^{2} t\right)\left(1+\cos ^{2} t\right)}+\frac{2}{t^{3}}
\end{aligned}
$$


Clearly, Theorem 2.9 cannot be applied to equation (3.4). In this case Corollary 3.5 is applicable. By Corollary 3.5, we obtain

$$
\begin{aligned}
& \limsup _{t \rightarrow \infty} \frac{1}{t^{\lambda-1}} \int_{t_{1}}^{t}\left(s-t_{1}\right)^{\lambda}\left[\phi(s)-\frac{1}{4} r(s) v(s)\left(\frac{\lambda}{s-t_{1}}-\frac{q(s)}{r(s)}\right)^{2}\right] \mathrm{d} s \\
= & \limsup _{t \rightarrow \infty} \frac{1}{t} \int_{t_{1}}^{t}\left(s-t_{1}\right)^{2}\left[\frac{\left(1+\sin ^{2} s\right)}{s\left(4+\sin ^{2} s\right)\left(1+\cos ^{2} s\right)}+\frac{2}{s^{3}}-\frac{1}{4 s^{3}} \frac{\left(3 s-t_{1}\right)^{2}}{\left(s-t_{1}\right)^{2}}\right] \mathrm{d} s=\infty
\end{aligned}
$$

and

$$
\begin{aligned}
& \limsup _{t \rightarrow \infty} \frac{1}{t} \int_{t_{1}}^{t}(t-s)^{\lambda}\left[\phi(s)-\frac{1}{4} r(s) v(s)\left(\frac{\lambda}{t-s}-\frac{q(s)}{r(s)}\right)^{2}\right] \mathrm{d} s \\
= & \limsup _{t \rightarrow \infty} \frac{1}{t} \int_{t_{1}}^{t}(t-s)^{2}\left[\frac{1+\sin ^{2} s}{s\left(4+\sin ^{2} s\right)\left(1+\cos ^{2} s\right)}+\frac{2}{s^{3}}-\frac{1}{4 s^{3}}\left(\frac{s+t}{t-s}\right)^{2}\right] \mathrm{d} s=\infty .
\end{aligned}
$$

Thus by Corollary 3.5, every solution of equation (3.4) is oscillatory. Moreover, $y=\sin t$ is an oscillatory solution of (3.4).

\section{Numerical results}

We have obtained oscillatory solutions of the above equations numerically under different initial conditions. The first three figures represents the solutions and its derivative of the first example and the solutions of the second example is given in the last three figures.

\section{Disfocality and disconjugacy}

In this section, we assume that $y(t)$ is a nontrivial solution of (1.1) with $y(a)=0=y(b), y(t) \neq 0$ for $t \in(a, b)$. Then there exists a $c \in(a, b)$ such that $y^{\prime}(c)=0$.

Definition 4.1. Equation (1.1) is said to be right-disfocal in $[c, b)$ if the solution of $(1.1)$ with $y^{\prime}(c)=0, y(c) \neq 0$ do not have zero in $[c, b)$.

Equation (1.1) is said to be left-disfocal in $(a, c]$ if the solution of (1.1) with $y^{\prime}(c)=0, y(c) \neq 0$, do not have zeros in $(a, c]$.

Equation (1.1) is said to be disconjugate in $(a, b)$, if no non trivial solution of (1.1) has more than one zero in $(a, b)$. 
Figure-1.1
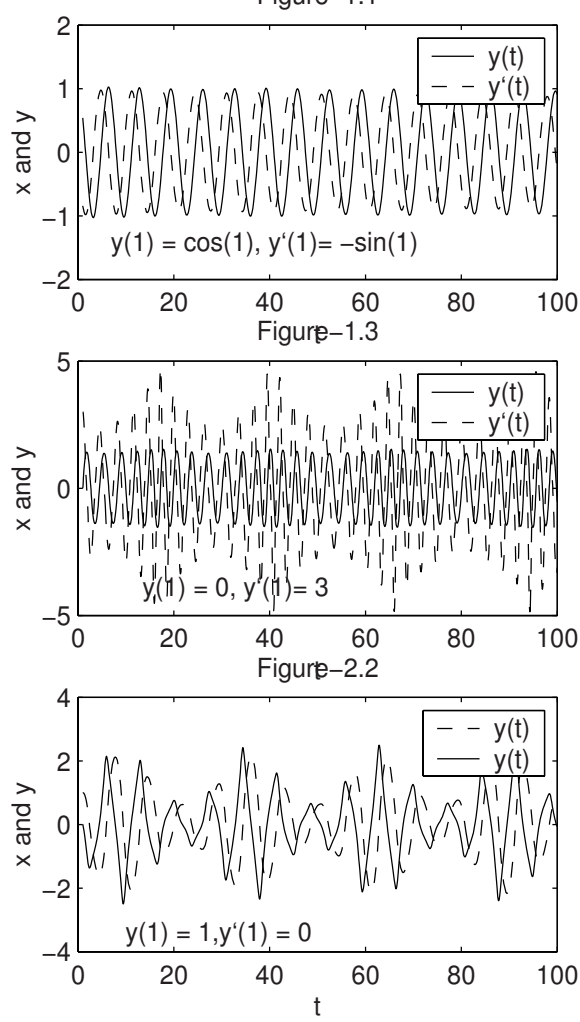

Figure-1.2
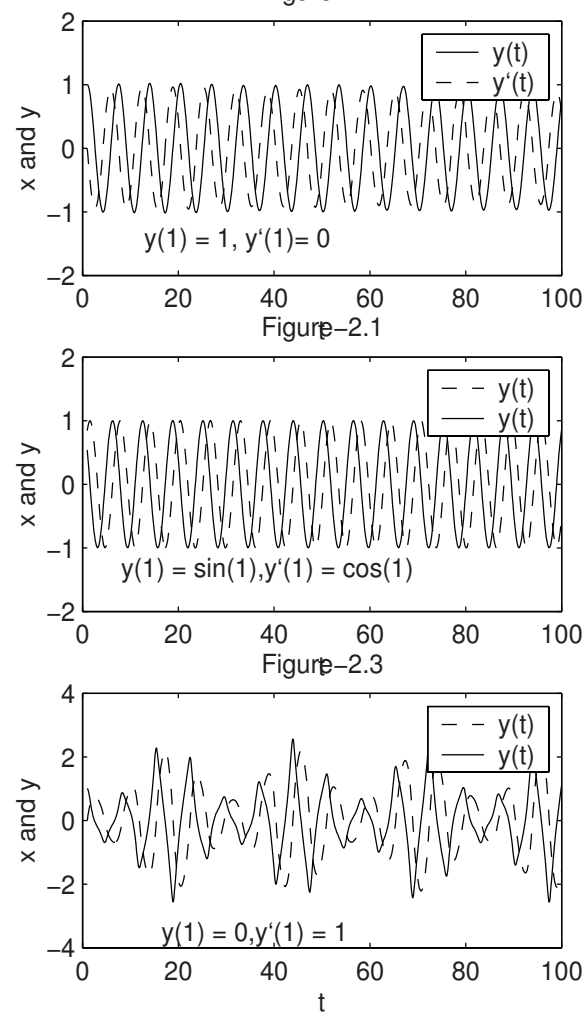

FiguRE 1. Figure for oscillaton

\section{TheOREM 4.2.}

(i) Assume that equation(1.1) is right disfocal in $[c, b)$. Then for any $H \in X$ and $C^{1}\left(\left[t_{0}, \infty\right),(0, \infty)\right)$, and $\kappa \in C^{1}\left[t_{0}, \infty\right)$

$$
\begin{aligned}
& \int_{c}^{b} H(b, s) \phi(s) \mathrm{d} s \\
\leq & H(b, c) v(c) r(c) \kappa(c)+\frac{1}{4 \mu} \int_{c}^{b} r(s) v(s)\left[h_{2}(b, s)+\frac{q(s)}{r(s)} \sqrt{H}(b, s)\right]^{2} \mathrm{~d} s,
\end{aligned}
$$

where

$$
v(t)=\exp \left(-2 \mu \int_{t_{0}}^{t} \kappa(s) \mathrm{d} s\right)
$$




\section{OSCILLATION CRITERIA FOR DIFFERENTIAL EQUATIONS OF SECOND ORDER}

and

$$
\phi(t)=v(t)\left\{L p(t)-q(t) \kappa(t)+\mu r(t) \kappa^{2}(t)-(r(t) \kappa(t))^{\prime}\right\} .
$$

(ii) Assume that equation(1.1) is left disfocal in (a,c]. Then for any $H \in X$ and $\kappa \in C^{1}\left(\left[t_{0}, \infty\right),(0, \infty)\right)$,

$$
\begin{aligned}
& \int_{a}^{c} H(s, a) \phi(s) \mathrm{d} s \\
\leq & -H(c, a) v(c) r(c) \kappa(c)+\frac{1}{4 \mu} \int_{a}^{c} r(s) v(s)\left[h_{1}(s, a)-\frac{q(s)}{r(s)} \sqrt{H}(s, a)\right]^{2} \mathrm{~d} s .
\end{aligned}
$$

(iii) Assume that equation (1.1) is disconjugate on $(a, b)$. Then for any $H \in X$ there exists $c \in(a, b)$ such that (4.1) or (4.2) hold.

Proof.

(i) Let $y$ be a solution of (1.1) satisfying $y^{\prime}(c)=0$ and $y(c)>0$. Since (1.1) is right disfocal in $[c, b)$, then $y(t)>0$ on $[c, b)$. So that $u(t)$ defined by $(2.3)$ exists on $[c, b)$ and $u(c)=v(c) r(c) \kappa(c)$. Then required inequalities follows from Theorem 2.2.

(ii) Let $y$ be a solution of (1.1) satisfying $y^{\prime}(c)=0$ and $y(c)>0$. Since (1.1) is left disfocal in $(a, c]$, then $y(t)>0$ on $(a, c]$. So that $u(t)$ defined by $(2.3)$ exists on $(a, c]$ and $u(c)=v(c) r(c) \kappa(c)$. Then the required inequality follows from Theorem 2.2.

(iii) Suppose that $(1.1)$ is disconjugate on $(a, b)$. Let $y(t)$ be a solution of (1.1) with $y^{\prime}(c)=0, y(c) \neq 0$, where $c \in(a, b)$. Then $y(t)$ has at most one zero in $(a, c]$ or one zero in $[c, b)$. Hence (1.1) is left disfocal in $(a, c]$ or right disfocal in $[c, b)$. Hence $(4.1)$ or $(4.2)$ holds.

\section{Remark 4.3.}

(i) If for some $H \in X$ and $\kappa \in C^{1}\left(\left[t_{0}, \infty\right),(0, \infty)\right)$ such that

$$
\begin{aligned}
& \int_{c}^{b} H(b, s) \phi(s) \mathrm{d} s \\
>H(b, c) v(c) r(c) \kappa(c)+\frac{1}{4 \mu} \int_{c}^{b} r(s) v(s)\left[h_{2}(b, s)+\frac{q(s)}{r(s)} \sqrt{H}(b, s)\right]^{2} \mathrm{~d} s, &
\end{aligned}
$$

then (1.1) is not right disfocal in $[c, b)$. 
(ii) If for some $H \in X$ and $\kappa \in C^{1}\left(\left[t_{0}, \infty\right),(0, \infty)\right)$ such that

$$
\begin{aligned}
& \int_{a}^{c} H(s, a) \phi(s) \mathrm{d} s \\
& >-H(c, a) v(c) r(c) \kappa(c)+\frac{1}{4 \mu} \int_{a}^{c} r(s) v(s)\left[h_{1}(s, a)-\frac{q(s)}{r(s)} \sqrt{H}(s, a)\right]^{2} \mathrm{~d} s,
\end{aligned}
$$

then (1.1) is not left disfocal in $(a, c]$.

(iii) If for some $H \in X$ and $\kappa \in C^{1}\left(\left[t_{0}, \infty\right),(0, \infty)\right)$ such that

$$
\begin{aligned}
& \int_{a}^{c} H(s, a) \phi(s) \mathrm{d} s \\
>-H(c, a) v(c) r(c) \kappa(c)+\frac{1}{4 \mu} \int_{a}^{c} r(s) v(s)\left[h_{1}(s, a)-\frac{q(s)}{r(s)} \sqrt{H}(s, a)\right]^{2} \mathrm{~d} s, &
\end{aligned}
$$

and

$$
\begin{aligned}
& \int_{c}^{b} H(b, s) \phi(s) \mathrm{d} s \\
>H(b, c) v(c) r(c) \kappa(c)+\frac{1}{4 \mu} \int_{c}^{b} r(s) v(s)\left[h_{2}(b, s)+\frac{q(s)}{r(s)} \sqrt{H}(b, s)\right]^{2} \mathrm{~d} s, &
\end{aligned}
$$

holds. Then (1.1) is not disconjugate in $(a, b)$.

TheOREM 4.4. Assume that for some $H \in X$, there exists $c \in(a, b)$ such that

$$
\begin{aligned}
& \int_{a}^{c} H(s, a) \phi(s) \mathrm{d} s \\
>-H(c, a) v(c) r(c) \kappa(c)+\frac{1}{4 \mu} \int_{a}^{c} r(s) v(s)\left[h_{1}(s, a)-\frac{q(s)}{r(s)} \sqrt{H}(s, a)\right]^{2} \mathrm{~d} s &
\end{aligned}
$$

and

$$
\begin{aligned}
& \int_{c}^{b} H(b, s) \phi(s) \mathrm{d} s \\
> & H(b, c) v(c) r(c) \kappa(c)+\frac{1}{4 \mu} \int_{c}^{b} r(s) v(s)\left[h_{2}(b, s)+\frac{q(s)}{r(s)} \sqrt{H}(b, s)\right]^{2} \mathrm{~d} s
\end{aligned}
$$

then every solution of equation(1.1) has at least two zeros in $(a, b)$.

P r o o f. If above two inequalities hold, then the equation (1.1) is not left disfocal in $(a, c]$ and the equation (1.1) is not right disfocal in $[c, b)$, which implies that is 


\section{OSCILLATION CRITERIA FOR DIFFERENTIAL EQUATIONS OF SECOND ORDER}

not disconjugate in $(a, b)$. Hence there exists a solution with two zeros in $(a, b)$. Hence the proof of the theorem is complete.

Acknowledgement. The authors are grateful to the referees for their valuable comments and suggestions.

\section{REFERENCES}

[1] BURTON, T. A.-GRIMER, R.: Stability properties of $\left(r u^{\prime}\right)^{\prime}+a f(u) g\left(u^{\prime}\right)=0$, Monatsh. Math. 74 (1970), 211-222.

[2] CASSELL, J. S.: The assymptotic behaviour of a class of linear oscillatore, Quart. J. Math. Oxford Ser. (3) 32 (1981), 287-302.

[3] COPPEL, W. A.: Stability and Asymptotic Behaviour of Differential Equations, Heath, Boston, 1965.

[4] EL-SAYED, M. A. An oscillation criterion for a forced second order linear differential equations, Proc. Amer. Math. Soc. 118 (1993), 813-817.

[5] GRACE, S. R.-LALLI, B. S.: An oscillation criterion for second order strongly sublinear differential equations, J. Math. Anal. Appl. 123 (1987), 584-588.

[6] GRAEF, J. R.-SPIKES, P. W.: Asymptotic behaviour of solution of a second order nonlinear differential equations, J. Differential Equations 17 (1975), 451-476.

[7] GRAEF, J. R.-SPIKES, P. W.: Boundedness and convergence to zero of solutions of a forced second order nonlinear differential equations, J. Math. Anal. Appl. 62 (1978), 295-309.

[8] HARDY, G. H.-LITTLEWOOD, J. E.-POLYA, G.: Inequalities, Cambridge University Press, Cambridge, 1988.

[9] HUANG, C. C.: Oscillation and nonoscillation for second order linear differential equations, J. Math. Anal. Appl. 210 (1997), 712-723.

[10] KARTSATOUS, A. G.: Maintenance of oscillations under the effect of periodic forcing term, Proc. Amer. Math. Soc. 33 (1972), 377-383.

[11] KEENER, M. S.: Solutions of a certain linear homogeneous second order differential equations, Appl. Anal. 1 (1971), 57-63.

[12] KONG, Q.: Interval criteria for oscillation of second order linear ordinary differential equations, J. Math. Anal. Appl. 229 (1999), 258-270.

[13] LALLI, B. S.: On boundedness of solutions of certain second order differential equations, J. Math. Anal. Appl. 25 (1969), 182-188.

[14] LEIGHTON, W.: Comparison theorems for linear differential equations of second order, Proc. Amer. Math. Soc. 13 (1962), 603-610.

[15] LI, W. T.: Oscillation of certain second-order nonlinear differential equations, J. Math. Anal. Appl. 217 (1998), 1-14.

[16] LI, W. T.-AGARWAL, R. P.: Interval oscillation criteria related to integral averaging technique for certain nonlinear differential equations, J. Math. Anal. Appl. 245 (2000), $171-188$.

[17] LI, W. T.-AGARWAL, R. P.: Interval oscillation criteria for second order nonlinear differential equations with damping, Comput. Math. Appl. 40 (2000), 217-230.

[18] LI, W. T.-AGARWAL, R. P.: Interval oscillation criteria for a forced nonlinear ordinary differential equations, Appl. Anal. 75 (2000), 341-347.

[19] LI, W. T.-AGARWAL, R. P.: Interval oscillation criteria for second order forced nonlinear differential equations with damping, Panamer. Math. J. 11 (2001), 109-117. 


\title{
A. K. NANDAKUMARAN - S. PANIGRAHI
}

[20] LI, W. T.-ZHANG, M. Y.-FEI, X. L.: Oscillation criteria for a second order nonlinear differential equation with damping term, Indian J. Pure Appl. Math. 30 (1999), 1017-1029.

[21] PARHI, N.-PANIGRAHI, S.: Disfocality and Liapunov type inequalies for third order equations, Appl. Math. Lett. 16 (2003), 227-233.

[22] RAGOVCHENKO, Y. V.: Oscillation criteria for certain nonlinear differential equations, J. Math. Anal. Appl. 229 (1999), 399-416.

[23] RAINKEIN, S. M.: Oscillation theorems for second order nonhomogeneous linear differential equations, J. Math. Anal. Appl. 53 (1976), 550-553.

[24] SKIDMORE, A.-LEIGHTON, W.: On the equation $y^{\prime \prime}+p(x) y=f(x)$, J. Math. Anal. Appl. 43 (1973), 46-55.

[25] SKIDMORE, A.-BOWERS, J. J.: Oscillatory behaviour of solutions of $y^{\prime \prime}+p(x) y=$ $f(x)$, J. Math. Anal. Appl. 49 (1975), 317-323.

[26] TEUFEL, H.: Forced second order nonlinear oscillations, J. Math. Anal. Appl. 40 (1972), $148-152$.

[27] WONG, J. S.: Oscillation criteria for a forced second order linear differential eqations, J. Math. Anal. Appl. 231 (1999), 235-240.

[28] WONG, J. S.-BURTON, T. A.: Some properties of solution of $u^{\prime \prime}+a(t) f(u) g\left(u^{\prime}\right)=0$, Monatsh. Math. 69 (1965), 364-374.

[29] UTZ, W. R.: Properties of solutions of $u^{\prime \prime}+g(t) u^{2 n-1}=0$, Monatsh. Math. 66 (1962), $56-60$.

Received 31. 7. 2007

\author{
Department of Mathematics \\ Indian Institute of Science \\ Bangalore 560 012 \\ INDIA \\ E-mail: panigrahi2008@gmail.com \\ spsm@uohyd.ernet.in
}

